\title{
High Wolbachia infection rate in four-eyed fir bark beetle (Polygraphus proximus) populations of Tomsk province
}

\author{
R. Bykov ${ }^{1 *}$, I. Kerchev ${ }^{2}$, M. Yudina ${ }^{1,3}$, G. Yurlova ${ }^{1}$, A. Tikhomirova ${ }^{3}$, Yu. Ilinsky ${ }^{1,3}$ \\ ${ }^{1}$ Institute of Cytology and Genetics SB RAS, Novosibirsk, Russia \\ ${ }^{2}$ Institute of Systematics and Ecology of Animals SB RAS, Novosibirsk, Russia \\ ${ }^{3}$ Novosibirsk State University, Novosibirsk, Russia \\ *e-mail:bykovra@bionet.nsc.ru
}

Key words: Polygraphus proximus, Wolbachia, pest, Siberia

Motivation and Aim: The four-eyed fir bark beetle (Polygraphus proximus) is invasive pest of the Siberian fir [1]. Studying of its biology, in particular associations with any bacterial symbionts, may be important for biological control of this pest. Bacteria of the genus Wolbachia are common symbionts of many insects [2]. In some cases, these bacteria play significant role in host biology. This is the first study of Wolbachia infection in $P$. proximus populations.

Methods and Algorithms: The collection includes 152 samples of $P$. proximus from five regions of Tomsk province. Total DNA was individually extracted from whole beetles. Wolbachia detection was performed by PCR with primers specific to two housekeeping loci of Wolbachia. Infection rates were estimated for each studied locality. The multilocus sequence typing approach was performed to characterize Wolbachia isolates.

Results: Wolbachia symbiont was found in all studied localities of Tomsk province, in particular in Chainsky (44 \%), Molchanovsky (42\%), Bakcharsky (23\%), Krivosheinsky (77\%) and Tomsky (51\%) regions. The average infection rate was $47 \%$ (95\% confidence interval, $39-56 \%)$.

Conclusion: Here we firstly report on Wolbachia infection in P. proximus. Our data suggests of high Wolbachia infection rate in the populations of four-eyed fir bark beetle of Tomsk province. Further analysis of Wolbachia-P. proximus interactions may be used to develop new approach to control four-eyed fir bark beetle populations.

Acknowledgements: Supported by the RFBR (18-316-00099) and by the RSF (15-1410014).

\section{References}

1. Kerchev I.A. (2014) Ecology of four-eyed fir bark beetle Polygraphus proximus Blandford (Coleoptera; Curculionidae, Scolytinae) in the West Siberian region of invasion. Russian Journal of Biological Invasions. 5(3):176-185.

2. Zug R., Hammerstein P. (2012) Still a host of hosts for Wolbachia: analysis of recent data suggests that $40 \%$ of terrestrial arthropod species are infected. PloS One. 7(6):e38544. 